\title{
Determinates of Regain in Body Mass Index among Malnourished Aids Patients on Therapeutic Food in Amhara National Regional State, Northwest Ethiopia: A Retrospective Cohort Study
}

\author{
Molla Gedefaw ${ }^{1 *}$, Moges Tariku² \\ ${ }^{1}$ GAMBY College of Medical Sciences, Bahir Dar, Ethiopia \\ ${ }^{2}$ USAID, Amhara Region, Ethiopia \\ Email: mollagedefaw@yahoo.com
}

Received 26 March 2015; accepted 5 May 2015; published 12 May 2015

Copyright (C) 2015 by authors and Scientific Research Publishing Inc. This work is licensed under the Creative Commons Attribution International License (CC BY). http://creativecommons.org/licenses/by/4.0/

\section{Abstract}

Although its utilization is greatly disputed, ready-to-use therapeutic food has been started as clinical nutrition care and treatment for malnourished adult AIDS patients since 2011 in Amhara National Regional State. However, factors determining the intended outcome (weight gain) have not been properly investigated. The main objective of the study was to assess improvement in body mass index and to identify its determinant factors. A retrospective cohort study design was conducted. Cluster sampling was employed to select health facilities in which the service was provided in the region. Of the 44 health facilities, nine were selected using lottery method, and all patients receiving the care in these health institutions were included in the study. Using tailored structured checklist, data were collected, organized and cleaned. Using paired T-test existence of difference between the mean of body mass index at admission and at $3^{\text {rd }}$ visit was measured. Finally analysis of association between some selected independent variables with the outcome variable was done using logistic regression model at $95 \% \mathrm{CI}$ and $\mathrm{p}<0.05$. Of 431 study participants, $175(40.6 \%)$ study participants' body mass index was improved $\left(\geq 18.5 \mathrm{~kg} / \mathrm{m}^{2}\right)$. Paired T-test revealed that there was a statistically significant difference between mean of body mass index at admission and at the $3^{\text {rd }}$ visit. Good ready-to-use therapeutic food treatment adherence (AOR 11.145; 95\% CI 6.556, 18.946), moderate acute malnutrition at admission (AOR 6.71; 95\% CI $2.618,17.195$ ), good ART adherence (AOR 2.136; 95\% CI, 1.269, 3.595) and being male (AOR 1.73; 95\% CI 1.052, 2.850) have a statistically significant contribution for body mass index improve-

"Corresponding author. 
ment. The study identifies factors that determine gain in body mass index among AIDS patients on ready-to-use therapeutic food. However, although the study revealed a statistically significant difference between body mass index during enrolment, and after three months, we disagreed with the right and left utilization of imported food because of lack of sustainability, and aid dependency. We rather recommend interventions that encourage households to produce food with similar outcomes from locally available food staff.

\section{Keywords}

Therapeutic Food, Body Mass Index, Regain, AIDS, Ethiopia

\section{Background}

Ethiopia is one of the Sub-Saharan African Countries which are highly affected by HIV/AIDS for the last three decades. Currently in Ethiopia, there are about 789,960 adult populations living with HIV/AIDS. From these, 204,000 (38.7\%) are found in Amhara National Regional State. In addition, at National level, there are about 398,686 HIV positive people who need ART. Out of these, the share of Amhara National Regional State is 99,032 (40\%) [1].

Weight loss is common in HIV/AIDS patients. HIV gradually weakens the immune system and harms nutritional status through the reduction of intake, absorption and use of nutrients, and increased metabolism needs. Malnutrition can in turn aggravate the effects of HIV by increasing vulnerability to AIDS-related illnesses [2] [3]. That means malnutrition and HIV work in a vicious cycle. The weakening of the immune system because of HIV can lead to malnutrition, and malnutrition weakens the immune system of HIV positive people, contributing to rapid progression to AIDS [4].

Cognizant of this fact, numerous steps have been taken over the last 20 years to improve the long-term management of HIV infection in developing countries, resulting in better immune function, reduced mortality, and continued survival. However, experiences reveal that malnutrition plays a pivotal role in the success of HIV AIDS care. That means that malnutrition drastically decreases success of treatment outcomes [5]. Studies indicated that patients who had severe acute malnutrition were 4 to 5 times more likely to die in the first 90 days after starting ART. Likewise, patients with moderate acute malnutrition are 2 to 3 times more likely to die in the first 90 days after starting ART [6]. There are many research reports about the benefit of ready-to-use therapeutic food especially for children in severe malnutrition in different African countries. There are also experiences on its use in African Adults AIDS patients [7]. This longitudinal study revealed that in nearly 50\% of the patents on therapeutic food the treatment did not achieve its objectives. They also identified factors that contributed for its failure. Of them, nutritional status of patients at enrolment, sex of the patient, and whether patients was on ART or not yet on ART. Maldey et al. (2014) [8] reported on outcomes of patients on RUFT in Ethiopian patients. According to this study sex, educational status of patients, nutritional status of patients at admission, and presence of opportunistic infection were important predictors of outcomes of RUTF.

Although success stories about the importance of ready-to-use therapeutic food have been reported here and there, and are recommended by the World Health Organization, and other authoritative organizations such as UNICEF, its right and left utilization for the treatment of malnutrition has been facing serious critics [9]. Lathaman and his colleagues published a 24-page commentary in the Journal of the World Public Health Nutrition Association. In this article, they clearly and openly elucidated the merits of commercially produced ready-to-use therapeutic food (RUTF), when to use, and demerits of such commercially produced ready-to-use therapeutic, and its abuses with emphasis to its promotion even with WHO, and UNICEF. Moreover, they clearly had shown in this article that there was no standardized guideline for its use, and its possible health hazards including contamination with killing germs. They condemned the right and left utilization of commercially produced RUTF including its use for the prevention of malnutrition to the extent that such utilization could undermine exclusive breast feeding in children.

Despite these, Ethiopia has started clinical nutrition care and support program for adult AIDs patients since 2011 in Amhara National Regional State in 44 health facilities. Plump nut produce of France was the type of 
RUTF used in Ethiopia. However, studies are lacking which identify factors associated with regain in body mass index among patients receiving this care especially in Amhara National Regional State. Therefore, this study is timely, and findings of the study can have theoretical, as well as practical significance.

\section{Methodology}

\subsection{Study Design, Area and Period}

Retrospective cohort study design was applied. The study was conducted from January 1/2012 to December $31 / 2012$.

Study setting: Amhara National Regional Sate is one of the largest regions in Ethiopia. A total of 44 health institutions are available where ART and RUTF are administered. Of these, nine health institutions were randomly selected.

Target population: All adult AIDS patients who are on ART and RUTF in 44 Health Institutions of Amhara National Regional State.

Study population: All HIV/AIDS patients who were receiving HIV/AIDS care in the nine selected health institutions of Amhara National Regional Sate.

\subsection{Sampling Procedure}

Cluster sampling method was applied. By considering the homogeneity of the study participants, nine health facilities were selected by simple random sampling from 44 health facilities in which RUTF was applied. The selected health institutions were the following: D/Markos Health Center, Finote Selam Hospital, Dangila Health Center, Bahir Dar Health Center, Azezo Health Center, Shegaw-Motta Rural Hospital, Woldia Health Center, Dessie Regional Referral Hospital and D/Birhan Zonal Referral Hospital. Then all patients who received HIV/AIDS care at the nine cluster health institutions on RUTF were taken.

Inclusion/exclusion criteria: All adult AIDS patients who were on RUTF who were non pregnant were included in the study.

Data collection tool: Data was collected using tailored structured checklist developed for the study.

Data collection procedure: Records of all AIDS patients were made available to data collectors in all the nine health facilities. Data was collected by diploma level trained health professionals who collected data from recoded files of each patient who fulfilled the inclusion criteria.

Data analysis: The collected data was manually checked for completeness, coded, and entered into a computer, and analyzed using SPSS version 16 for windows. Bivariate, and multivariate logistics regression were fitted. Possible association was measured using adjusted odds ratio at 95\% confidence interval. To know whether there is a statistically significant difference between BMI at enrolment, and at the end of the $3^{\text {rd }}$ month of treatment, paired t-test was performed.

Quality assurance: To maintain the quality of the data appropriate data collection tool was prepared based on the registered data, data collectors and supervisors were trained how to collect the data, and how to maintain the quality of the data. Two data collectors and one supervisor were assigned at each health facility. The collected data were checked on daily bases by supervisors for completeness and consistency. Finally, appropriate statistical analysis techniques were applied.

\section{Results}

Table 1 displayed socio-demographic characteristics of study participants. As displayed in the table, a total of 431 records of patients fulfilled the predetermined criteria. Of these, 279 (65\%) of them were females. More than half of study participants were taken from health centers. Around 336 (78\%) study participants were urban dwellers. About 370 (86\%) of the study participant were Orthodox Christian. Around 194 (45\%) of the study participants were married and nearly 349 (81\%) of them had less than 4 house hold size. Nearly $70 \%$ of patients were between the age group of 25 to 44 years of age.

Table 2 displays the characteristics of the study participants at nutrition rehabilitation program admission. Of 431 study participants, 131 (30.4\%) had opportunistic infection (OI) at the time of admission to the program. In addition, 348 (80.7\%) study participants were moderate acute malnutrition (MAM) and the remaining, 83 (19.3\%) were severe acute malnutrition (SAM) at the time of admission to the program. 
Table 1. The socio-demographic characteristics of the study participants in Amhara National Regional State $(n=431)$.

\begin{tabular}{|c|c|c|}
\hline Variables & Frequency & $\%$ \\
\hline \multicolumn{3}{|l|}{ Sex } \\
\hline Female & 279 & 64.7 \\
\hline Male & 152 & 35.3 \\
\hline \multicolumn{3}{|l|}{ Age } \\
\hline$\geq 24$ & 38 & 8.8 \\
\hline $25-34$ & 161 & 37.4 \\
\hline $35-44$ & 140 & 32.5 \\
\hline $45-54$ & 63 & 14.6 \\
\hline $55-64$ & 19 & 4.4 \\
\hline $65+$ & 10 & 2.3 \\
\hline \multicolumn{3}{|l|}{ Marital status } \\
\hline Married & 194 & 45 \\
\hline Never married & 65 & 15 \\
\hline Separated & 31 & 7.2 \\
\hline Divorced & 105 & 24.4 \\
\hline Widow/widower & 36 & 8.4 \\
\hline \multicolumn{3}{|l|}{ Religion } \\
\hline Orthodox & 370 & 65.8 \\
\hline Others & 61 & 14.2 \\
\hline \multicolumn{3}{|l|}{ Educational status } \\
\hline Illiterate & 208 & 48.3 \\
\hline Primary & 105 & 24.3 \\
\hline Secondary \& above & 118 & 27.4 \\
\hline
\end{tabular}

Table 2. Selected attributes of patients at the time of admission to nutrition rehabilitation program in Amhara National Regional State $(n=431)$.

\begin{tabular}{|c|c|c|}
\hline Characteristics & Frequency & $\%$ \\
\hline \multicolumn{3}{|l|}{ ART adherence } \\
\hline Good & 284 & 65.9 \\
\hline Poor & 147 & 34.1 \\
\hline \multicolumn{3}{|l|}{ OI } \\
\hline Yes & 131 & 30.4 \\
\hline No & 300 & 69.6 \\
\hline \multicolumn{3}{|l|}{ Nutritional status } \\
\hline MAM & 348 & 80.7 \\
\hline SAM & 83 & 19.3 \\
\hline
\end{tabular}


Figure 1 depicts BMI at during enrolment to RUTF and after three months of treatment. As shown in the figure, mean BMI at enrolment, and after three months of RUTF treatment was at $16.87 \mathrm{~kg} / \mathrm{m}^{2} \pm 1.27$, and $17.99 \pm$ 1.58 , respectively. Paired t-test revealed that there was a statistically significant difference between the initial BMI and BMI at the $3^{\text {rd }}$ month. We took BMI instead of weight for statistical analysis in order to compare it with other similar studies. We did this because any change in body mass index of adults is the result of change in weight, and cannot be a change in height.

Table 3 displays determinants of regain in BMI. Male patients were nearly 2 times more likely achieve normal BMI status compared with females [AOR 1.731; 95\% CI (1.052, 2.850)]. Those patients who adhered to RUTF treatment were 11 times more likely to achieve normal BMI compared to those who did not adhered to the RUTF treatment [AOR 11.145; 95\% CI $(6.556,18.946)]$. Those patients who were moderately malnourished were 7 times more likely to achieve the desired BMI compared with those who were severely malnourished at the time of enrolment [AOR 6.71; 95\% CI $(2.618,17.195)]$.

Moreover, those patients who adhered to Antiretroviral treatment (ART) were 2 times more likely to regain the desired BMI compared to those who did not adhered [2.136; 95\% CI (1.269, 3.595)].

Table 3. The association of BMI improvement with some selected variables in Amhara National Regional State $(n=431)$.

\begin{tabular}{|c|c|c|c|c|c|}
\hline \multirow{3}{*}{ Variables } & \multicolumn{2}{|c|}{ BMI improved } & \multirow{3}{*}{ COR $(95 \%$ CI $)$} & \multirow{3}{*}{ AOR (95\% CI) } & \multirow{3}{*}{$\mathrm{p}$ value } \\
\hline & Yes & No & & & \\
\hline & No (\%) & No (\%) & & & \\
\hline
\end{tabular}

Sex

$$
\begin{aligned}
& \text { Female } \\
& \text { Male }
\end{aligned}
$$

Adherence to RUTF treatment

Good

Poor

151 (35)

24 (5.6)

102 (23.7)

73 (16.9)

$177(41.1)$

79 (18.3)

81 (18.8)

13.59 (8.21, 22.52)

$11.15(6.56,18.95)$

0.000

175 (40.6)

Nutritional status at admission

MAM

SAM

ART adherence status

Good

Poor

Educational status

Secondary \& above

$76(17.6)$

57 (13.2)

$132(30.6)$

$61(14.2)$

$31(7.2)$

144 (33.4)

100 (23.2)

156 (36.2)

$68(15.8)$

18 (4.2)

157 (36.4)

$12.12(5.14,28.55)$

1

$2.2(1.44,3.38)$

1

$2.14(1.25,3.6)$

0.004

1

1

$1.623(1.026,2.566) \quad 1.533(0.844,2.785)$

0.161

OI at admission

No

OI after admission

Yes

No

$157(36.4)$

$\mathrm{COR}=$ crude odds ratio; $\mathrm{AOR}=$ adjusted odds ratio. 


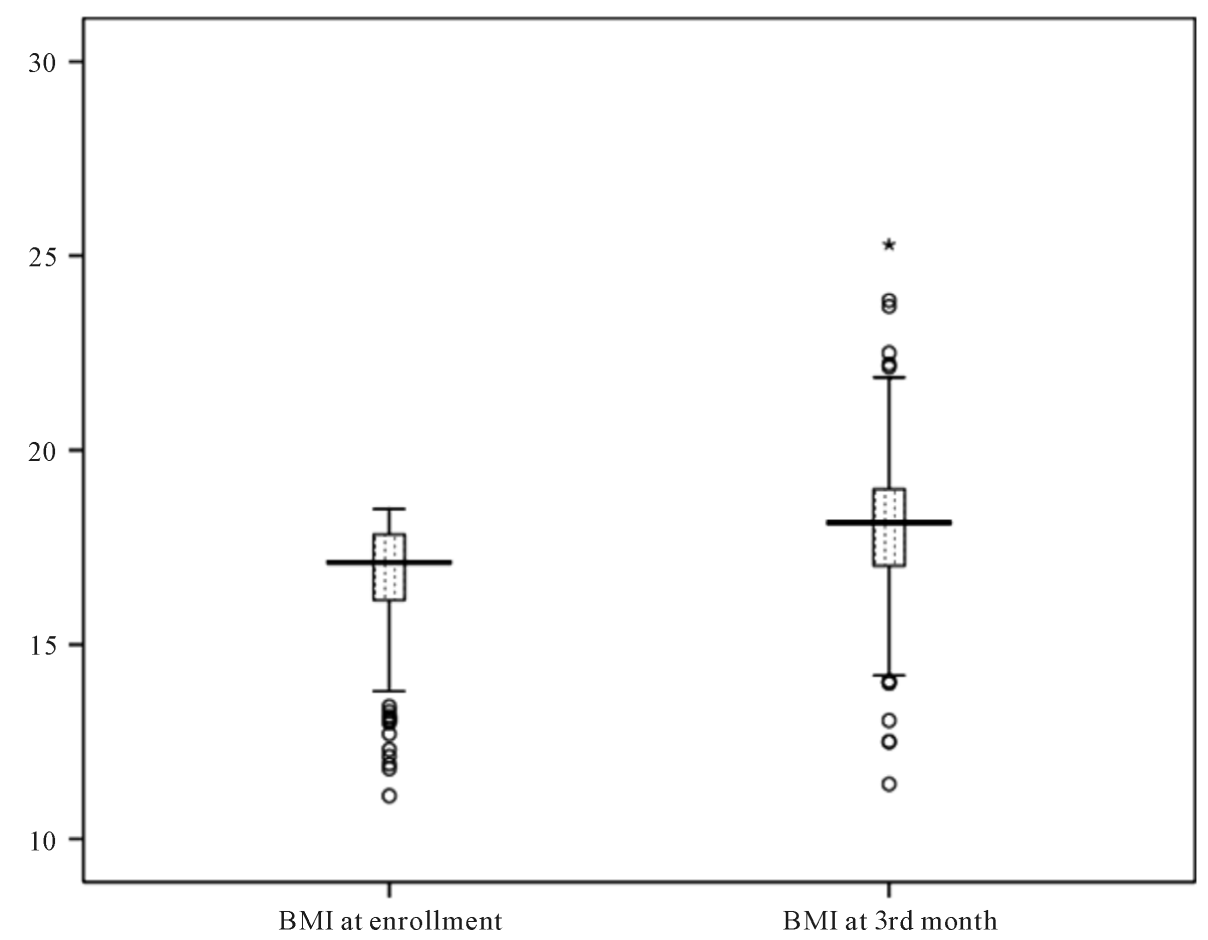

Figure 1. Body Mass Index of study participants at enrolment for RUFT, and after three months of follow up in Amhara national Regional State.

\section{Discussions}

The finding of this study shows that about $41 \%$ of patients achieved normal BMI after three months of treatment. This figure was $84 \%$ in a Zambian study [10]. The graduation rate in Zambia was two folds of the graduation rate in Amhara National Regional State. This difference may be caused by the difference in socio-demographic HIV/AIDS patients' characteristics, clinicians' skill of treatment and health facilities set up between Malawi and Amhara National Regional State. According to the Zambian health protocol, greater than $75 \%$ of discharges should be cured, less than $10 \%$ of discharges should be deaths, and less than $15 \%$ of discharges should be defaulters [10]. In Ethiopia, there are no written indicators to measure the program effectiveness.

Another study which was conducted in Kenya and Uganda showed that $47.4 \%$ of clients BMI improved from the total number of clients treated by RUFT [11]. It is a bit better than the prevalence of BMI improvement among HIV/AIDS patients in Amhara National Regional State. Again the difference may be caused by the difference in socio-demographic HIV/AIDS patients' characteristics, clinicians' skill of treatment $\&$ health facilities set up in Kenya and Uganda as compared with Amhara National Regional State.

The finding of this study shows that males have better chance of BMI improvement than females. The odds of BMI improvement of males is nearly 2 times as compared with the chance of BMI improvement of females. This finding is in line with a study done in the northern Ethiopia [8].

The finding of this study also shows that ART adherence status is another important determinant factor for BMI Improvement of malnourished HIV/AIDS patients who were treated by RUTF. This finding is in line with a study conducted in Malawi [12]. This could be partly explained by the fact that good ART adherence reduces viral loads, increase CD4, disease progression delayed, the occurrence of OI reduced \& the total health outcome increases [12].

In addition this study showed that moderately malnourished patients have better chance of BMI improvement than SAM clients do. This is also in agreement with a study conducted in Zambia [10].

Finally, the findings of this study showed that the odds of BMI improvement of clients who have good RUTF treatment adherence is near to 11 times higher than lost follow up clients. This finding is in accordance with a study finding done in Malawi [12].

It has been noted that almost all studies recommended the utilization of commercially produced RUTF by 
considering its clear advantages such as long shelf life, and well known components. However, its disadvantage in relation to sustainability, cost, and dependency seem to be completely forgotten. The commentary by Latham MC et al., 2011 [9] should be seriously considered.

The finding of the study could have been even better had it been possible to employ prospective cohort study design which enables researchers to exhaustively include all potentials determinant variables. However, the variables captured for this study were pertinent. Hence, this study was able to show the proportion of patients who achieved normal body mass index by regaining weight within three months treatment by RUTF, and identified practically relevant variables which are amenable to change.

\section{Conclusion}

The study identifies factors that determine gain in body mass index among AIDS patients on ready-to-use therapeutic food. However, although the study revealed a statistically significant difference between body mass index during enrolment, and after three months, we disagreed with the right and left utilization of imported food because of lack of sustainability, and aid dependency. We rather recommend interventions that encourage households to produce food with similar outcomes from locally available food staff.

\section{References}

[1] Ethiopian Demographic and Health Survey (2011) Final Report. Addis Ababa, Ethiopian.

[2] ACC/SCN (2004) Managing Successful Nutrition Programmes, ACC/SCN State-of-the-Art Series, Nutrition Policy Discussion Paper No. 8. ACC/SCN, Geneva.

[3] (2005) World Health Organization Consultation on Nutrition and HIV/AIDS in Africa, April 10-13, 2005. Participants' Statement, Durban.

[4] Batterham, M.J. (2005) Investigating Heterogeneity in Studies of Resting Energy Expenditure in Persons with HIV/ AIDS: A Meta-Analysis 1-3. American Journal of Clinical Nutrition, 81, 702-713.

[5] Consay and Kennedy (1999) Infection and Malnutrition Cycle. ACC/SCN.

[6] Paton, N.I.S., Earnest, S.A. and Bellamy, R. (2006) The Impact of Malnutrition on Survival and the CD4 Count Response in HIV-Infected Patients Starting Antiretroviral Therapy. HIV Medicine, 7, 323-330. http://dx.doi.org/10.1111/j.1468-1293.2006.00383.x

[7] Ahoua, L., Umutoni, C., et al. (2011) Nutrition Outcomes of HIV-Infected Malnourished Adults' Treated with Readyto-Use Therapeutic Food in Sub-Saharan Africa: A Longitudinal Study. Journal of the International AIDS Society, 14, 2. http://dx.doi.org/10.1186/1758-2652-14-2

[8] Maldey, B., Haile, F. and Shumye, A. (2014) Outcome of Ready to Use Food Therapy among Patients on HIV/AIDS Care in Mekelle Hospital, Northern Ethiopia: Retrospective Cohort Study. Journal of AIDS Clinical Research, 5, 1-6.

[9] Latham, M.C., Jonsson, U., Sterken, E. and Kent, G. (2011) RUTF Stuff: Can the Children Be Saved with Fortified Peanut Paste? World Nutrition, 2, 62-85.

[10] Food and Nutritional Technical Assistance II Project (FANTA-2) (2010) The Analysis of the Nutrition Situation in Uganda \& Kenya. FANTA-2, AED, Washington DC.

[11] Food and Nutrition Technical Assistance Project (2008) Ethiopia Nutrition and HIV Tools. FANTA, Washington DC.

[12] Anawani, G. and Navario, P. (2005) Nutrition and AIDS in Sub Saharan Africa. An Overview. Nutrition, 21, 96-99. http://dx.doi.org/10.1016/j.nut.2004.09.013 